\title{
BAYESIAN PARAMETER ESTIMATION IN IMAGE RECONSTRUCTION FROM SUBSAMPLED BLURRED OBSERVATIONS
}

\author{
Miguel Vega ${ }^{a}$, Javier Mateos $^{b}$, Rafael Molina $^{b}$ and Aggelos K. Katsaggelos ${ }^{c *}$
}

a) Dpto. de Lenguajes y Sistemas Informáticos, Universidad de Granada, 18071 Granada, Spain

b) Dpto. de Ciencias de la Computación e I.A., Universidad de Granada, 18071 Granada, Spain

c) Dept. of Electrical and Computer Engineering, Northwestern University, Evanston, IL 60208-3118

\begin{abstract}
In this paper we consider the estimation of the unknown hyperparameters for the problem of reconstructing a high-resolution image from multiple undersampled, shifted, blurred and degraded frames with subpixel displacement errors. We derive mathematical expressions for the iterative calculation of the maximum likelihood estimate (mle) of the unknown hyperparameters given the low resolution observed images. Finally, the proposed method is tested on a synthetic image.
\end{abstract}

\section{INTRODUCTION}

Over the last two decades research has been devoted to the problem of reconstructing a high-resolution image from multiple undersampled, shifted by subpixel displacement, blurred and degraded frames. However, as reported in [1], not much work has been devoted to the efficient calculation of the reconstruction or the estimation of the associated hyperparameters.

In our previous work $[2,3,4]$ the general framework for frequency domain multi-channel signal processing developed in [5] and [6] was used to provide estimates of the hyperparameter in high resolution reconstruction from subsampled images. In this paper our approach is extended to deal with the presence of blurring in the observation model, thus making the method suitable for applications such as remote sensing and Astronomy, where blurred images are usually captured.

The rest of the paper is organized as follows. The problem formulation is described in section 2. Section 3 describes the high resolution prior image model and the process to obtain the low resolution images from the high resolution one. The application of the Bayesian paradigm to calculate the maximum a posteriori (MAP) high resolution image and to estimate the hyperparameters is described in section 4. Experimental results are described in section 5. Finally, section 6 concludes the paper.

\section{PROBLEM FORMULATION}

Consider a sensor array with $L_{1} \times L_{2}$ sensors, each sensor having $N_{1} \times N_{2}$ pixels with size of each sensing element $T_{1} \times T_{2}$. Our aim is to reconstruct an $M_{1} \times M_{2}$ high resolution image, with $M_{1}=L_{1} \times N_{1}$ and $M_{2}=L_{2} \times N_{2}$, from $L_{1} \times L_{2}$ low-resolution observed images of size $N_{1} \times N_{2}$.

*This work has been partially supported by the "Comisión Nacional de Ciencia y Tecnología" under contract TIC2000-1275.
Note that in order for our objective to make sense we need to assume that the original high-resolution scene is bandlimited to wavenumbers $L_{1} /\left(2 T_{1}\right)$ and $L_{2} /\left(2 T_{2}\right)$ along the horizontal and vertical directions, respectively. To maintain the aspect ratio of the reconstructed image we consider the case where $L_{1}=L_{2}=L$.

In the ideal case, the low resolution sensors are shifted with respect to each other by a value proportional to $T_{1} / L \times T_{2} / L$ (note that if the sensors are shifted by values proportional to $T_{1} \times T_{2}$ the high-resolution image reconstruction problem becomes singular). However, in practice there can be small perturbations around those ideal locations. Thus, for $l_{1}, l_{2}=0, \ldots, L-1$, the horizontal and vertical displacements $d_{l 1, l 2}^{x}$ and $d_{l 1, l 2}^{y}$ of the $\left[l_{1}, l_{2}\right]$-th sensor with respect to the $[0,0]$-th reference sensor are given by

$$
d_{l 1, l 2}^{x}=\frac{T_{1}}{L}\left(l_{1}+\epsilon_{l 1, l 2}^{x}\right) \text { and } d_{l 1, l 2}^{y}=\frac{T_{2}}{L}\left(l_{2}+\epsilon_{l 1, l 2}^{y}\right),
$$

where $\epsilon_{l 1, l 2}^{x}$ and $\epsilon_{l 1, l 2}^{y}$ denote respectively the normalized horizontal and vertical displacement errors. We assume that $\left|\epsilon_{l 1, l 2}^{x}\right|<1 / 2$ and $\left|\epsilon_{l 1, l 2}^{y}\right|<1 / 2$ with $\epsilon_{0,0}^{x}=\epsilon_{0,0}^{y}=0$. The normalized horizontal and vertical displacement may be assumed to be known (see $[7,8]$ for details) or estimated previously to the reconstruction process (see $[9,10]$ ). In [11] we can find an approach where the displacements are assumed unknown and are estimated simultaneously with the high-resolution image.

\section{IMAGE AND DEGRADATION MODELS}

Let $\mathbf{f}$ be the $\left(M_{1} \times M_{2}\right) \times 1$ high resolution image and $\mathbf{g}_{l 1, l 2}$ the $\left(N_{1} \times N_{2}\right) \times 1$ observed low resolution image from the $(l 1, l 2)$ sensor, $(l 1, l 2) \in\{0, \ldots L-1\}^{2}$. Our goal is to reconstruct $\mathbf{f}$ from $\left\{\mathbf{g}_{l 1, l 2} \mid(l 1, l 2) \in\{0, \ldots L-1\}^{2}\right\}$. In order to apply the Bayesian paradigm to this problem we define next our image and high to low degradation models.

\subsection{Image Model}

Our prior knowledge about the smoothness of the object luminosity distribution makes it possible to model the distribution of $\mathbf{f}$ by a simultaneous autoregression (SAR):

$$
p(\mathbf{f} \mid \alpha)=\frac{1}{Z_{\text {prior }}(\alpha)} \exp \left\{-\frac{1}{2} \alpha \mathbf{f}^{t} \mathbf{C}^{t} \mathbf{C} \mathbf{f}\right\}
$$

where the parameter $\alpha$ measures the smoothness of the 'true' image,

$$
Z_{\text {prior }}(\alpha)=\left(\prod_{i, j} \lambda_{i j}^{2}\right)^{-1 / 2}(2 \pi / \alpha)^{\left(M_{1} \times M_{2}\right) / 2},
$$


where $\lambda_{i j}=1-2 \phi\left(\cos \left(2 \pi i / M_{1}\right)+\cos \left(2 \pi j / M_{2}\right)\right)$, for $i=$ $1,2, \ldots, M_{1}, j=1,2, \ldots, M_{2}$, and $\mathbf{C}$ is the Laplacian operator.

\subsection{Model for obtaining the low-resolution observed images}

The process to obtain the observed low resolution image by the $(l 1, l 2)$ sensor, $\mathbf{g}_{l 1, l 2}$, from $\mathbf{f}$ can be modeled in two stages as follows.

In the first stage, the optical distortion in the capture process is considered, obtaining $\mathbf{f}^{l 1, l 2}$. This image represents a blurred version of the original high-resolution one, according to

$$
\mathbf{f}^{l 1, l 2}=\mathbf{B}_{l 1, l 2} \mathbf{f},
$$

where $\mathbf{B}_{l 1, l 2}$ is a $\left(M_{1} \times M_{2}\right) \times\left(M_{1} \times M_{2}\right)$ matrix that defines the systematic blur of the $(l 1, l 2)$ sensor, assumed to be known and approximated by a block circulant matrix.

The second stage models the CCD pixel resolution. Let $\mathbf{H}_{l 1, l 2}$ be an $\left(M_{1} \times M_{2}\right) \times\left(M_{1} \times M_{2}\right)$ integrating matrix that may have different forms. In [12] $h_{l}^{i}$ has the form

$$
h_{l}^{i}(u)=\left\{\begin{array}{ll}
\frac{1}{L} & u=-(L-1), \ldots, 0 \\
0 & \text { otherwise }
\end{array} .\right.
$$

Note that in this case, $h_{l 1}^{1}=h_{l 2}^{2}, \forall i, \epsilon_{l}^{i}=0$, the normalized horizontal and vertical displacement errors in Eq. (1) satisfy $\epsilon_{l 1, l 2}^{x}=\epsilon_{l 1, l 2}^{y}=0$ and $\mathbf{H}_{l 1, l 2}=\mathbf{H}, \forall l 1, l 2=0, \ldots, L-1$.

Let $\mathbf{D}_{l 1}$ and $\mathbf{D}_{l 2}$ now be the 1-D downsampling matrices defined by

$$
\mathbf{D}_{l 1}=\mathbf{I}_{N_{1}} \otimes \mathbf{e}_{l}^{t}, \quad \mathbf{D}_{l 2}=\mathbf{I}_{N_{2}} \otimes \mathbf{e}_{l}^{t},
$$

where $\mathbf{I}_{N_{i}}$ is the $N_{i} \times N_{i}$ identity matrix, $\mathbf{e}_{l}$ is the $L \times 1$ unit vector whose nonzero element is in the $l$-th position, $\otimes$ denotes the Kronecker product operator. Then for each sensor the discrete low-resolution observed image $\mathbf{g}_{l 1, l 2}$ can be written as

$$
\mathbf{g}_{l 1, l 2}=\mathbf{D}_{l 1, l 2} \mathbf{H}_{l 1, l 2} \mathbf{B}_{l 1, l 2} \mathbf{f}+\mathbf{v}_{l 1, l 2},
$$

where $\mathbf{D}_{l 1, l 2}=\mathbf{D}_{l 1} \otimes \mathbf{D}_{l 2}$, denotes the $\left(N_{1} \times N_{2}\right) \times\left(M_{1} \times M_{2}\right)$ $2 \mathrm{D}$ downsampling matrix and $\mathbf{v}_{l 1, l 2}$ is modeled as independent white noise with variance $\beta_{l 1, l 2}^{-1}$.

If $\mathbf{W}_{l 1, l 2}$ denotes the $\left(N_{1} \times N_{2}\right) \times\left(M_{1} \times M_{2}\right)$ matrix $\mathbf{W}_{l 1, l 2}=$ $\mathbf{D}_{l 1, l 2} \mathbf{H}_{l 1, l 2} \mathbf{B}_{l 1, l 2}$, then we have

$$
\begin{aligned}
& p\left(\mathbf{g}_{l 1, l 2} \mid \mathbf{f}, \beta_{l 1, l 2}\right) \propto \frac{1}{Z\left(\beta_{l 1, l 2}\right)} \\
& \quad \times \quad \exp \left[-\frac{\beta_{l 1, l 2}}{2}\left\|\mathbf{g}_{l 1, l 2}-\mathbf{W}_{l 1, l 2} \mathbf{f}\right\|^{2}\right],
\end{aligned}
$$

where $Z\left(\beta_{l 1, l 2}\right)=\left(2 \pi / \beta_{l 1, l 2}\right)^{\left(N_{1} \times N_{2}\right) / 2}$. We denote by $\mathbf{g}$ the sum of the upsampled low resolution images, that is,

$$
\mathbf{g}=\sum_{u=0}^{L-1} \sum_{v=0}^{L-1} \mathbf{D}_{u, v}^{t} \mathbf{g}_{u, v}
$$

Then

$$
\begin{aligned}
& p(\mathbf{g} \mid \mathbf{f}, \underline{\beta}) \propto \frac{1}{Z_{\text {noise }}(\underline{\beta})} \\
& \quad \times \quad \exp \left[-\frac{1}{2} \sum_{l 1=0}^{L-1} \sum_{l 2=0}^{L-1} \beta_{l 1, l 2}\left\|\mathbf{g}_{l 1, l 2}-\mathbf{W}_{l 1, l 2} \mathbf{f}\right\|^{2}\right]
\end{aligned}
$$

where $\beta=\left(\beta_{l 1, l 2} \mid(l 1, l 2) \in\{0, \ldots, L-1\}^{2}\right)$, and $Z_{\text {noise }}(\beta)=$ $\prod_{l 1=0}^{L-1} \prod_{l 2=0}^{L-1} Z\left(\beta_{l 1, l 2}\right)$.

Note that the main difference between the model in Eq. (9) and the one used in [2] and [3] is the presence of additional blurring in the observation process. We will examine in the coming section how the additional blurring matrix can be dealt with using blocksemicirculant (BSC) matrices (see [5] and [6]).

\section{BAYESIAN ANALYSIS}

The steps we follow in this paper to estimate the hyperparameters, $\alpha$ and $\beta$, and the original image are

\section{Step I: Estimation of the hyperparameters}

The hyperparameters $\hat{\alpha}$ and $\hat{\beta}=\left(\hat{\beta}_{l 1, l 2} \mid(l 1, l 2) \in\{0, \ldots, L-\right.$ $\left.1\}^{2}\right)$ are first selected as

$$
\hat{\alpha}, \underline{\hat{\beta}}=\arg \max _{\alpha, \underline{\beta}} \mathcal{L}_{\mathbf{g}}(\alpha, \underline{\beta})=\arg \max _{\alpha, \underline{\beta}} \log p(\mathbf{g} \mid \alpha, \underline{\beta}),
$$

where $p(\mathbf{g} \mid \alpha, \underline{\beta})=\int_{\mathbf{f}} p(\mathbf{f} \mid \alpha) p(\mathbf{g} \mid \mathbf{f}, \underline{\beta}) d \mathbf{f}$.

\section{Step II: Estimation of the original image}

Once the hyperparameters have been estimated, the estimation of the original image, $\mathbf{f}_{(\hat{\alpha}, \hat{\beta})}$, is selected as the image which minimizes

$$
M(\mathbf{f}, \mathbf{g} \mid \hat{\alpha}, \underline{\hat{\beta}})=\hat{\alpha}\|\mathbf{C f}\|^{2}+\sum_{l 1=0}^{L-1} \sum_{l 2=0}^{L-1} \hat{\beta}_{l 1, l 2}\left\|\mathbf{g}_{l 1, l 2}-\mathbf{W}_{l 1, l 2} \mathbf{f}\right\|^{2},
$$

so, we have

$$
\mathbf{f}_{(\hat{\alpha}, \underline{\hat{\beta}})}=\mathbf{Q}(\hat{\alpha}, \underline{\hat{\beta}})^{-1} \sum_{l 1=0}^{L-1} \sum_{l 2=0}^{L-1} \hat{\beta}_{l 1, l 2} \mathbf{W}_{l 1, l 2}^{t} \mathbf{g}_{l 1, l 2},
$$

where $\mathbf{Q}(\hat{\alpha}, \hat{\beta})=\hat{\alpha} \mathbf{C}^{t} \mathbf{C}+\sum_{l 1, l 2=0}^{L-1} \hat{\beta}_{l 1, l 2} \mathbf{W}_{l 1, l 2}^{t} \mathbf{W}_{l 1, l 2}$.

Note that we are using maximum likelihood for estimating the hyperparameter and maximum a posteriori (MAP) for estimating the high resolution image. Furthermore, although steps I and II are separated, the iterative scheme to be proposed performs both estimations simultaneously.

The estimation process we are using could be performed within the so called hierarchical Bayesian approach [13] by including hyperpriors on the unknown hyperparameter $\hat{\alpha}$ and hypervector $\hat{\beta}$. However, the possibility of incorporating additional knowledge on them by means of gamma or other distributions will not be discussed here (see [13]).

Let us examine the estimation process in detail. Fixing $\alpha$ and $\underline{\beta}$ and expanding the function $M(\mathbf{f}, \mathbf{g} \mid \alpha, \underline{\beta})$ around $\mathbf{f}_{(\alpha, \beta)}$, we have

$$
\begin{aligned}
M(\mathbf{f}, \mathbf{g} \mid \alpha, \underline{\beta})= & M\left(\mathbf{f}_{(\alpha, \underline{\beta})}, \mathbf{g} \mid \alpha, \underline{\beta}\right) \\
& +\frac{1}{2}\left(\mathbf{f}-\mathbf{f}_{(\alpha, \underline{\beta})}\right)^{t} \mathbf{Q}(\alpha, \underline{\beta})\left(\mathbf{f}-\mathbf{f}_{(\alpha, \underline{\beta})}\right) .
\end{aligned}
$$

Therefore

$$
p(\mathbf{g} \mid \alpha, \underline{\beta})=\frac{\exp \left\{-M\left(\mathbf{f}_{(\alpha, \underline{\beta})}, \mathbf{g} \mid \alpha, \underline{\beta}\right) / 2\right\}|\mathbf{Q}(\alpha, \underline{\beta})|^{-1 / 2}}{Z_{\text {prior }}(\alpha) Z_{\text {noise }}(\underline{\beta})} .
$$


Differentiating $-2 \mathcal{L}_{\mathbf{g}}(\alpha, \beta)$ with respect to $\alpha$ and $\beta$ so as to find the conditions satisfied at the maxima, we have

$$
\begin{aligned}
& \left\|\mathbf{C f}_{(\alpha, \underline{\beta})}\right\|^{2}+\operatorname{tr}\left[\mathbf{Q}(\alpha, \underline{\beta})^{-1} \mathbf{C}^{t} \mathbf{C}\right]=\frac{M_{1} \times M_{2}}{\alpha} \\
& \left\|\mathbf{g}_{l 1, l 2}-\mathbf{W}_{l 1, l 2} \mathbf{f}_{(\alpha, \underline{\beta})}\right\|^{2}+\operatorname{tr}\left[\mathbf{Q}(\alpha, \underline{\beta})^{-1} \mathbf{W}_{l 1, l 2}^{t} \mathbf{W}_{l 1, l 2}\right] \\
& \quad=\frac{N_{1} \times N_{2}}{\beta_{l 1, l 2}}, \text { for } l 1, l 2=0, \ldots, L-1 .
\end{aligned}
$$

We will use the following algorithm for the estimation of the hyperparameters and the high-resolution image

1. Choose $\alpha^{0}$ and $\beta^{0}$.

2. Compute $\mathbf{f}_{\left(\alpha^{0}, \underline{\beta}^{0}\right)}$ using (11) with $\hat{\alpha}=\alpha^{0}, \underline{\hat{\beta}}=\underline{\beta}^{0}$.

3. Repeat for $k=1,2, \ldots$

i) Calculate $\alpha^{k}$ and $\underline{\beta}^{k}$ by substituting $\alpha^{k-1}$ and $\underline{\beta}^{k-1}$ in the left hand side of (12) and (13).

ii) Compute $\mathbf{f}_{\left(\alpha^{k}, \underline{\beta}^{k}\right)}$ by (11) with $\hat{\alpha}=\alpha^{k}, \underline{\hat{\beta}}=\underline{\beta}^{k}$

until $\left\|\mathbf{f}_{\left(\alpha^{k}, \beta^{k}\right)}-\mathbf{f}_{\left(\alpha^{\left.k-1, \beta^{k-1}\right)}\right.}\right\|$ is less than a prescribed bound.

It is important to note that for the problem of Bayesian parameter estimation in image reconstruction from subsampled blurred observations all the calculations involve BSC matrices, allowing us to work in the frequency domain, applying the framework developed at [5] and [6].

Equations (12) and (13) can be also obtained with the EMalgorithm [14] with $\mathcal{X}^{t}=\left(\mathbf{f}^{t}, \mathbf{g}^{t}\right)$ and $\mathcal{Y}=\mathbf{g}=\left[\begin{array}{ll}\mathbf{0} & \mathbf{I}\end{array}\right]^{\mathbf{t}} \mathcal{X}$ to iteratively increase $\mathcal{L}_{\mathbf{g}}(\alpha, \beta)$.

\section{EXPERIMENTAL RESULTS}

A number of simulations have been performed with the proposed algorithm over a set of images. Here we present results on the original image depicted at Fig. 1a. The criterion

$$
\left\|\mathbf{f}_{\left(\alpha^{k}, \underline{\beta}^{k}\right)}-\mathbf{f}_{\left(\alpha^{k-1}, \underline{\beta}^{k-1}\right)}\right\|^{2} /\left\|\mathbf{f}_{\left(\alpha^{k-1}, \underline{\beta}^{k-1}\right)}\right\|^{2}<10^{-6}
$$

was used for terminating iterations. We set $\mathbf{f}^{0}=\mathbf{g}$, where $\mathbf{g}$ has been defined in (8).

The performance of the restoration algorithms was evaluated by measuring the SNR improvement,

$$
\Delta_{S N R}=10 \times \log _{10}\left[\|\mathbf{f}-\mathbf{g}\|^{2} /\|\mathbf{f}-\hat{\mathbf{f}}\|^{2}\right],
$$

where $\mathbf{f}$ and $\hat{\mathbf{f}}$ are the original and estimated high resolution images, respectively.

According to (6) the original image, see Fig. 1b, was blurred by mean of an out-of-focus blur with radius 5 using Eq.(3). We then applied the integrating function described in Eq.(4) obtaining $\mathbf{u}=\mathbf{H B f}$. Then $\mathbf{u}$ was downsampled with $L_{1}=L_{2}=4$, thus obtaining 16 low resolution images, $u_{l 1, l 2}(x, y)=u\left(L_{1} x+\right.$ $\left.l 1, L_{2} y+l 2\right), x, y=0, \ldots, \frac{M_{1}}{4}-1, l 1, l 2=0, \ldots, 3$. Gaussian noise was added to each low resolution image to obtain three sets of sixteen low resolution images with 10, 20 and 30dB SNRs. The noise variances for each set of images are shown in table 1 . Figure 1a shows the obtained $\mathbf{g}_{00}$ for $30 \mathrm{~dB}$ SNR low resolution image. Figure 1c depicts the zero-order hold upsampled image $\mathrm{g}_{00}$ for $30 \mathrm{~dB}$ SNR. The initial image, $\mathbf{f}^{0}=\mathrm{g}$, for the $30 \mathrm{~dB}$ set according to (8) is shown in Fig. 1d and the estimated high-resolution a)

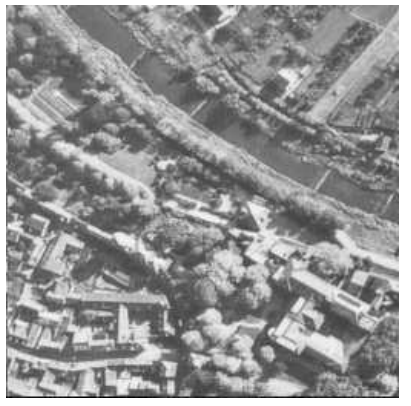

b)

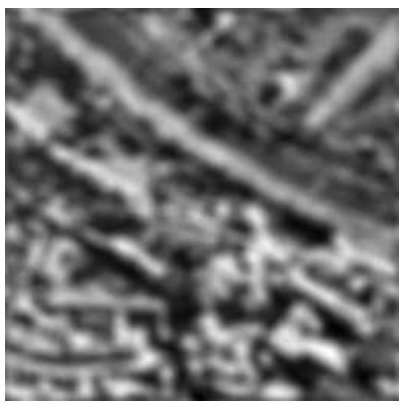

d)

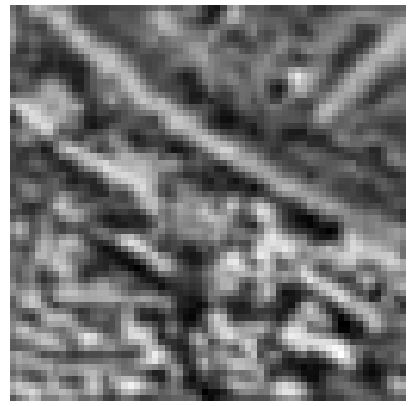

c)

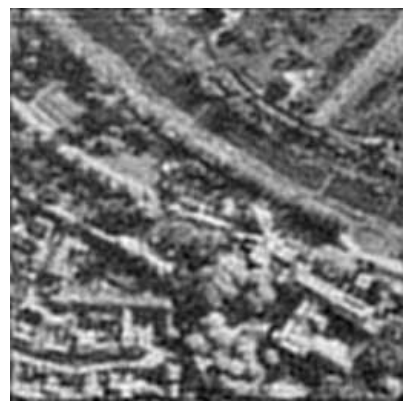

e)
Fig. 1. a) $30 \mathrm{~dB}$ SNR observation $\mathbf{g}_{00}$ from b) Original $256 \times$ 256 high-resolution image, c) zero-order hold for the $30 \mathrm{~dB}$ SNR observation $\mathbf{g}_{00}, \mathrm{~d}$ ) initial high resolution image and e) estimated high resolution image.

one in Fig. 1e. Visual inspection shows that the proposed method provides a clear improvement.

Numerical results for the three sets of images with SNRs of 10,20 and $30 \mathrm{~dB}$ are summarized in table 2 . It is clear that the proposed method improves the SNR even in the case of severe noise although higher improvements are obtained as the noise decreases. Each low resolution set of 16 observed images was bilinearly interpolated to obtain a $256 \times 256$ image. For all cases, the best results using bilinear interpolation are similar to the initial high resolution image. Table 2 also shows the number of iterations needed for the method to reach convergence according to the utilized criterion. For all sets of images no more than 22 iterations were needed. Each iteration took 15.5 seconds on a Pentium IV 1700.

Estimated image model parameters for the 10, 20 and $30 \mathrm{~dB}$ SNR sets of images were $\alpha^{-1}=208.67,196.40$ and 175.42 , respectively. The estimated noise variances for the low resolution images are presented in table 3. From this table we conclude that the proposed method produces accurate estimations of the low resolution image variances for all SNRs.

\section{CONCLUSIONS}

A new method to estimate the unknown hyperparameters in a image reconstruction problem from subsampled blurred observations 
Table 1. Noise variances for the low resolution image set with SNR of $10 \mathrm{~dB}, 20 \mathrm{db}$ and $30 \mathrm{db}$.

\begin{tabular}{|c|c|c|c|c|c|}
\hline & $\sigma_{l 1, l 2}^{2}$ & 0 & 1 & 2 & 3 \\
\hline $10 \mathrm{~dB}$ & 0 & 95.33 & 94.49 & 95.39 & 94.47 \\
& 1 & 94.93 & 95.12 & 94.89 & 94.85 \\
& 2 & 95.45 & 94.73 & 94.91 & 94.73 \\
& 3 & 94.94 & 94.87 & 94.47 & 95.28 \\
\hline $20 \mathrm{~dB}$ & 0 & 8.59 & 8.64 & 8.66 & 8.62 \\
& 1 & 8.61 & 8.64 & 8.60 & 8.60 \\
& 2 & 8.64 & 8.65 & 8.65 & 8.61 \\
& 3 & 8.65 & 8.64 & 8.62 & 8.60 \\
\hline $30 \mathrm{~dB}$ & 0 & 0.85 & 0.85 & 0.85 & 0.86 \\
& 1 & 0.86 & 0.86 & 0.85 & 0.85 \\
& 2 & 0.86 & 0.86 & 0.85 & 0.85 \\
& 3 & 0.85 & 0.86 & 0.85 & 0.86 \\
\hline
\end{tabular}

Table 2. Summary of results for the three different low resolution image sets.

\begin{tabular}{|c|c|c|c|}
\hline Low resolution SNR $(\mathrm{dB})$ & 10 & 20 & 30 \\
\hline Bilin. interp. $\Delta_{S N R}(\mathrm{~dB})$ & 0.06 & -0.14 & -0.17 \\
\hline Proposed alg. $\Delta_{S N R}(\mathrm{~dB})$ & 2.860 & 3.802 & 5.376 \\
\hline Iterations & 13 & 22 & 16 \\
\hline
\end{tabular}

has been proposed. Using BSC matrices all the matrix calculations involved in the hyperparameter maximum likelihood estimation can be performed in the Fourier domain. The approach followed can be used to assign the same hyperparameter to all low resolution image hyperparameters or to make them image dependent. The proposed method has been validated experimentally.

\section{REFERENCES}

[1] S. Borman and R. Stevenson, "Spatial resolution enhancement of low-resolution image sequences. A comprehensive review with directions for future research," Tech. Rep., Laboratory for Image and Signal Analysis, University of Notre Dame, 1998.

[2] R. Molina, M. Vega, J. Abad, and A. K. Katsaggelos, "Parameter estimation in bayesian high-resolution image reconstruction with multisensors," Submitted to IEEE Trans. Image Processing, 2002.

[3] J. Abad, M. Vega, R. Molina, and A. K. Katsaggelos, "Parameter estimation in super-resolution image reconstruction problems," in To appear at IEEE Conference on Acoustic, Speech and Signal Processing, 2003.

[4] J. Mateos, R. Molina, and A. K. Katsaggelos, "Bayesian high resolution image reconstruction with incomplete multisensor low resolution systems," in To appear at IEEE Conference on Acoustic, Speech and Signal Processing, 2003.

[5] A. K. Katsaggelos, K. T. Lay, and N. P. Galatsanos, "A general framework for frequency domain multi-channel signal processing," IEEE Trans. Image Processing, vol. 2, no. 3, pp. 417-420, 1993.
Table 3. Estimated noise variances for the $10 \mathrm{~dB}, 20 \mathrm{~dB}$ and $30 \mathrm{~dB}$ SNR low resolution image sets.

\begin{tabular}{|c|c|c|c|c|c|}
\hline & $\beta_{l 1, l 2}^{-1}$ & 0 & 1 & 2 & 3 \\
\hline $10 \mathrm{~dB}$ & 0 & 94.70 & 94.51 & 94.39 & 94.97 \\
& 1 & 91.16 & 94.99 & 93.61 & 94.62 \\
& 2 & 90.58 & 92.03 & 96.97 & 95.34 \\
& 3 & 94.26 & 96.01 & 91.98 & 95.07 \\
\hline $20 \mathrm{~dB}$ & 0 & 8.53 & 8.60 & 8.63 & 8.63 \\
& 1 & 8.24 & 8.62 & 8.47 & 8.56 \\
& 2 & 8.26 & 8.38 & 8.84 & 8.66 \\
& 3 & 8.55 & 8.81 & 8.41 & 8.55 \\
\hline $30 \mathrm{~dB}$ & 0 & 0.86 & 0.85 & 0.86 & 0.86 \\
& 1 & 0.82 & 0.85 & 0.85 & 0.85 \\
& 2 & 0.83 & 0.83 & 0.88 & 0.86 \\
& 3 & 0.85 & 0.88 & 0.84 & 0.85 \\
\hline
\end{tabular}

[6] M. R. Banham, N. P. Galatsanos, H. L. Gonzalez, and A. K. Katsaggelos, "Multichannel restoration of single channel images using a wavelet-based subband decomposition," IEEE Transactions on Image Processing, vol. 3, pp. 821-833, 1994.

[7] N. K. Bose and K. J. Boo, "High-resolution image reconstruction with multisensors," Int. Journ. Imaging Systems and Technology, vol. 9, pp. 141-163, 1998.

[8] M. K. Ng and A. M. Yip, "A fast MAP algorithm for highresolution image reconstruction with multisensors," Multidimensional Systems and Signal Processing, vol. 12, pp. 143164, 2001.

[9] H. Shekarforoush, M. Berthod, and J. Zerubia, "Subpixel image registration by estimating the polyphase decomposition of cross power spectrum," in Proceedings IEEE Conference on Computer Vision and Pattern Recognition, 1996, pp. 532537.

[10] A. Averbuch and Y. Keller, "Fft based image registration," in IEEE Conference on Audio, Speech and Signal Processing, 2002, vol. 4, pp. 3608-3611.

[11] B. C. Tom, N. P. Galatsanos, and A. K. Katsaggelos, "Reconstruction of a high resolution image from multiple low resolution images," in Super-Resolution Imaging, S. Chaudhuri, Ed., chapter 4, pp. 73-105. Kluwer Academic Publishers, 2001.

[12] D. Rajan and S. Chaudhuri, "An MRF-based approach to generation of super-resolution images from blurred observations," Journal of Mathematical Imaging and Vision, vol. 16, pp. 5-153, 2002.

[13] N. P. Galatsanos, V. Z. Mesarovic, R. Molina, A. K. Katsaggelos, and J. Mateos, "Hyperparameter estimation in image restoration problems with partially-known blurs," Optical Engineering, vol. 41, no. 8, pp. 1845-1854, 2002.

[14] A. P. Dempster, N. M. Laird, and D. B. Rubin, "Maximum likelihood from incomplete data," Journal of the Royal Statistics Society B, vol. 39, pp. 1-38, 1972. 